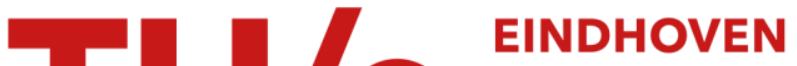

\section{Modified extended BDF time-integration methods, applied to circuit equations}

\section{Citation for published version (APA):}

Allaart-Bruin, S. M. A., Maten, ter, E. J. W., \& Verduyn Lunel, S. M. (2002). Modified extended BDF timeintegration methods, applied to circuit equations. (RANA : reports on applied and numerical analysis; Vol. 0225). Technische Universiteit Eindhoven.

\section{Document status and date:}

Published: 01/01/2002

\section{Document Version:}

Publisher's PDF, also known as Version of Record (includes final page, issue and volume numbers)

\section{Please check the document version of this publication:}

- A submitted manuscript is the version of the article upon submission and before peer-review. There can be important differences between the submitted version and the official published version of record. People interested in the research are advised to contact the author for the final version of the publication, or visit the $\mathrm{DOI}$ to the publisher's website.

- The final author version and the galley proof are versions of the publication after peer review.

- The final published version features the final layout of the paper including the volume, issue and page numbers.

Link to publication

\section{General rights}

Copyright and moral rights for the publications made accessible in the public portal are retained by the authors and/or other copyright owners and it is a condition of accessing publications that users recognise and abide by the legal requirements associated with these rights.

- Users may download and print one copy of any publication from the public portal for the purpose of private study or research.

- You may not further distribute the material or use it for any profit-making activity or commercial gain

- You may freely distribute the URL identifying the publication in the public portal.

If the publication is distributed under the terms of Article 25fa of the Dutch Copyright Act, indicated by the "Taverne" license above, please follow below link for the End User Agreement:

www.tue.nl/taverne

Take down policy

If you believe that this document breaches copyright please contact us at:

openaccess@tue.nl

providing details and we will investigate your claim. 


\title{
Modified Extended BDF Time-Integration Methods, Applied to Circuit Equations
}

\author{
Sandra Allaart-Bruin ${ }^{1}$, Jan ter Maten ${ }^{2}$, and Sjoerd Verduyn Lunel ${ }^{3}$ \\ 1 Eindhoven University of Technology, sbruin@win.tue.nl \\ 2 Eindhoven University of Technology, Philips Research Laboratories \\ 3 Leiden University
}

\begin{abstract}
Electric circuits designers are frequently interested in the transient behaviour of the designed circuit. A common method for time integration of the Differential Algebraic circuit Equations (DAE) is the Backward Differentiation Formula (BDF) method. In 1983, J. Cash proposed the Modified Extended BDF (MEBDF) method, which combines better stability properties and higher order of convergence than BDF, but requires more computations per step. We prove reduction of convergence order for MEBDF when applied to DAE's with higher DAE-index. However, because in practice, in circuit analysis, the DAE-index does not exceed 2, the reduction is quite moderate and it equals the BDF-order in that case. One gains better, or even unconditional, stability. One also obtains consistent solutions.
\end{abstract}

\section{Introduction}

In Circuit Design, Transient Analysis is most heavily used. There is constant interest in methods that offer better performance with respect to robustness as well as to reduction of CPU-time. Because the underlying circuit equations are Differential-Algebraic Equations (DAE), a point for robustness is how well the time integrator behaves when problems of higher DAE-index have to be treated: does reduction of order of convergence happen, and does one obtain consistent solutions. Other points of interest are stability conditions (see [8]), and the damping behavior along the imaginary axis.

Backward Differentiation Formula (BDF) Methods do not suffer from reduction of order of convergence and generate consistent solutions $[1,9,12]$, which made them very popular for circuit simulation, however at the cost of being conditionally stable when the order exceeds 2 . Improvements were looked for in combining BDF with Trapezoidal Rule [10] (less damping), or in new methods, like Implicit Runge-Kutta methods like Radau-methods [11] (3rd order L-stable Implicit Runge-Kutta methods with options for parallelism), or CHORAL (embedded method of order (2)3, stiffly accurate and L-stable)[7]. In this paper, we focus on Modified Extended BDF (MEBDF) methods [3] and we report on newly proven convergence order reduction when applied to DAE's of higher index and on the generation of consistent solutions. The MEBDF-methods offer better stability conditions than BDF. For instance, when applied to index-1 DAE's, the 3-step method has order 4 and is A-stable (see [9]). When applied to index-2 DAE's, the order of convergence reduces 
to 3 , but the method's stability property remains, which compares favorably to the 3-step BDF method. The MEBDF-methods easily fit a datastructure that was designed for an implementation of BDF-methods. Variants also allow parallelism [6].

We start with an explanation of the MEBDF method. After that we formulate two new theorems on the order of the MEBDF method. We continue with some numerical results of an example circuit and present results for a general test problem.

\section{Modified Extended BDF}

One timestep with the MEBDF method consists of three BDF steps and an evaluation step. This results in more work compared to BDF, but the order of convergence increases with one for most circuits. This implies that for convergence order 3 we normally apply the 3-step BDF method, while with the MEBDF method a 2-step method suffices. Well-posed circuits lead to an index-1 DAE. For this type of DAE's the k-step MEBDF has order $k+1$, while the k-step BDF has order $k$ (see Section 3 and [12]). Checking the circuit topology enables one roughly to distinguish between index-1 and index-2 problems [12]. Circuits that contain controlled elements require a much deeper analysis [5]. Several numerical methods suffer from convergence order reduction when applied to DAE's with higher index. For this reason, one is interested in time integration methods of which the order of convergence is robust with respect to the DAE-index quantity. A second property that one likes to see fulfilled is that the numerical solution satisfies the consistency property for DAE's: if one starts on the manifold defined by the algebraic constraints one wants to stay on it. An appreciated property would be that the method is able to generate a consistent solution even if one starts from an inconsistent initial value.

We consider the following quasi-linear DAE

$$
A \mathbf{x}^{\prime}(t)+\mathbf{g}(\mathbf{x}(t), t)=\mathbf{0}
$$

where $\mathbf{x}(t) \in D \subseteq \mathbb{R}^{m}, t \in I \subseteq \mathbb{R}$ and $A$ is a constant $m \times m$ matrix. MEBDF is defined by the following steps for integrating one timestep ${ }^{1}$.

- First BDF step: Solve for $\overline{\mathbf{x}}_{\ell}^{(\ell-k)_{2}}$ at $t_{\ell}$ by the $k$-step BDF-method

$$
A \sum_{i=0}^{k} \hat{\alpha}_{i} \mathbf{x}_{\ell-i}=-h \hat{\beta}_{k} \mathbf{g}\left(\mathbf{x}_{\ell}, t_{\ell}\right)
$$

\footnotetext{
${ }^{1}$ For occurring coefficients $\hat{\alpha}_{0}, \ldots \hat{\alpha}_{k}, \hat{\beta}_{k}, \alpha_{0}, \ldots, \alpha_{k}, \beta_{k}, \beta_{k+1}$ see $[2,3]$

${ }^{2} \cdot \ell$, is to denote that it is an approximation on time $t_{\ell} ;{ }^{-}$is to denote that it is a BDF solution; ${ }^{(\ell-k)}$ is to denote that it is computed in the $(\ell-k)$-th step.
} 
- Second BDF step: Compute an "extrapolated" solution at $t_{\ell+1}$ by BDF

$$
A \sum_{i=0}^{k} \hat{\alpha}_{i} \mathbf{x}_{\ell+1-i}=-h \hat{\beta}_{k} \mathbf{g}\left(\mathbf{x}_{\ell+1}, t_{\ell+1}\right),
$$

where $\mathbf{x}_{\ell}=\overline{\mathbf{x}}_{\ell}^{(\ell-k)}$. This solution is called $\overline{\mathbf{x}}_{\ell+1}^{(\ell-k)}$.

- Evaluation step: Compute

$$
\overline{\mathbf{g}}_{\ell}=\mathbf{g}\left(\overline{\mathbf{x}}_{\ell}^{(\ell-k)}, t_{\ell}\right), \quad \overline{\mathbf{g}}_{\ell+1}=\mathbf{g}\left(\overline{\mathbf{x}}_{\ell+1}^{(\ell-k)}, t_{\ell+1}\right) .
$$

- MEBDF step: Solve for a new solution $\mathbf{x}_{\ell}^{(\ell-k)}$ at $t_{\ell}$ :

$$
A \sum_{i=0}^{k} \alpha_{i} \mathbf{x}_{\ell-i}=-h \hat{\beta}_{k} \mathbf{g}\left(\mathbf{x}_{\ell}, t_{\ell}\right)-h\left(\beta_{k}-\hat{\beta}_{k}\right) \overline{\mathbf{g}}_{\ell}-h \beta_{k+1} \overline{\mathbf{g}}_{\ell+1} .
$$

For solving all equations we use a quasi-Newton method. This means that we don't compute a new Jacobian every iteration, but apply the same Jacobian as for the $k$-step BDF during the several iteration steps.

\section{Theoretical Results}

In this section we prove new results on the order of the MEBDF applied to DAE's. Here we restrict ourselves to a constant stepsize $h$, i.e. $t_{\ell+1}=t_{\ell}+h$ for all $\ell$. For $\ell \geq k$, the numerical solution $\left\{x_{\ell}\right\}$ of the $k$-step MEBDF applied to (1), satisfies

$$
A \frac{1}{h} \sum_{i=0}^{k} \alpha_{i} \mathbf{x}_{\ell-i}+\hat{\beta}_{k} \mathbf{g}\left(\mathbf{x}_{\ell}, t_{\ell}\right)+\left(\beta_{k}-\hat{\beta}_{k}\right) \overline{\mathbf{g}}_{\ell}+\beta_{k+1} \overline{\mathbf{g}}_{\ell+1}=\delta_{\ell} .
$$

Here, $\delta_{\ell}$ describes the perturbations in the $\ell$-th step for $\ell \geq k$, which are introduced by numerical computations including the errors arising from solving the nonlinear equations. Moreover, we introduce

$\mathbf{x}_{*}\left(t_{\ell}\right):=$ exact solution of $(1)$

$$
\begin{aligned}
\tilde{\mathbf{x}}_{\ell}: & =\mathbf{x}_{\ell}-\mathbf{x}_{*}\left(t_{\ell}\right), \quad \ell \geq 0, \\
\tau_{\ell}: & =A \frac{1}{h} \sum_{i=0}^{k} \alpha_{i} \mathbf{x}_{*}\left(t_{\ell-i}\right)+\beta_{k} \mathbf{g}\left(\mathbf{x}_{*}\left(t_{\ell}\right), t_{\ell}\right)+\beta_{k+1} \mathbf{g}\left(\mathbf{x}_{*}\left(t_{\ell+1}\right), t_{\ell+1}\right), \quad \ell \geq k .
\end{aligned}
$$

Here $\tau_{\ell}$ represents the local discretization error of the $k$-step MEBDF in the $\ell$-th step $(\ell \geq k)$. Now (2) may be written as

$$
A \frac{1}{h} \sum_{i=0}^{k} \alpha_{i} \tilde{\mathbf{x}}_{\ell-i}+B_{\ell} \tilde{\mathbf{x}}_{\ell}+\mathbf{h}\left(\tilde{\mathbf{x}}_{\ell}, t_{\ell}\right)+\tau_{\ell}-\delta_{\ell}-\nu_{\ell}-\tilde{\nu}_{\ell}=\mathbf{0},
$$


where

$$
\begin{aligned}
B_{\ell} & :=\hat{\beta}_{k} \mathbf{g}_{\mathbf{x}}\left(\mathbf{x}_{*}\left(t_{\ell}\right), t_{\ell}\right), \\
\mathbf{h}(\mathbf{x}, t) & :=\hat{\beta}_{k}\left[\mathbf{g}\left(\mathbf{x}+\mathbf{x}_{*}(t), t\right)-\mathbf{g}\left(\mathbf{x}_{*}(t), t\right)-\mathbf{g}_{\mathbf{x}}\left(\mathbf{x}_{*}(t), t\right) \mathbf{x}\right], \\
\nu_{\ell} & :=\left(\beta_{k}-\hat{\beta}_{k}\right)\left[\mathbf{g}\left(\mathbf{x}_{*}\left(t_{\ell}\right), t_{\ell}\right)-\overline{\mathbf{g}}_{\ell}\right], \\
\tilde{\nu}_{\ell} & :=\beta_{k+1}\left[\mathbf{g}\left(\mathbf{x}_{*}\left(t_{\ell+1}\right), t_{\ell+1}\right)-\overline{\mathbf{g}}_{\ell+1}\right] .
\end{aligned}
$$

\subsection{Index-1 DAE}

In this section, we assume that (1) is an index-1 DAE. This implies that the pair $\left\{A, B_{\ell}\right\}$ is index-1 tractable [12]. Let $Q$ be a projector onto $\operatorname{Ker}[A]$ and $P:=I-Q$. We define $G_{1 \ell}:=A+B_{\ell} Q$ and $\hat{\delta}_{\ell}$ by

$$
\hat{\delta}_{\ell}:= \begin{cases}Q G_{1 \ell}^{-1}\left(\delta_{\ell}+\nu_{\ell}+\tilde{\nu}_{\ell}\right), & \ell \geq k \\ Q G_{1 \ell}^{-1} \mathbf{h}\left(\tilde{\mathbf{x}}_{\ell}, t_{\ell}\right)+Q \tilde{\mathbf{x}}_{\ell}+Q G_{1 \ell}^{-1} B_{\ell} P \tilde{\mathbf{x}}_{\ell}, & \ell<k\end{cases}
$$

$\hat{\delta}_{\ell}$ represents the defect in the algebraic part for $\ell \geq k$. For the starting values, the corresponding defects are also described by $\hat{\delta}_{\ell}$.

Theorem 1. Suppose a constant $C_{1}>0$ exists such that the starting values satisfy the relation

$$
\left\|P \mathbf{x}_{\ell}-P \mathbf{x}_{*}\left(t_{\ell}\right)\right\| \leq C_{1} h^{k+1}, \quad \ell<k,
$$

and suppose constants $C_{2}$ and $C_{3}$ exist such that

$$
\left\|\delta_{\ell}\right\| \leq C_{2} h^{k}, \quad\left\|\hat{\delta}_{\ell}\right\| \leq C_{3} h^{k+1}, \quad \ell \geq k,
$$

Then a constant $C>0$ exists such that

$$
\left\|\mathbf{x}_{*}\left(t_{\ell}\right)-\mathbf{x}_{\ell}\right\| \leq C h^{k+1}, \quad \ell \geq 0 .
$$

Outline of the proof (for full details see [2]): We use the projectors $P$ and $Q$ to split the DAE into an algebraic part and a differential part. Next, we show the existence of a solution of the algebraic part. We use the inverse function theorem to show that this solution is locally unique. Substituting this solution into the differential part of the DAE results in an equation involving only index-1 variables. This equation can also be solved uniquely with the inverse function theorem. By applying the assumptions of the theorem we get the final result.

Remarks. Most well-posed circuits lead to an index-1 DAE [12]. For this type of DAE we conclude that the $k$-step MEBDF has order $k+1$ (cf. (6)), while the $k$-step BDF has order $k$. Note that the $k$-step MEBDF-methods are A-stable for $k \leq 3$, while for $\mathrm{BDF}$ this is restricted to the case $k \leq 2$ [3]. 


\subsection{Index-2 DAE}

Next, we assume that (1) is an index-2 DAE. Let $Q_{1 \ell}$ be a projector onto $\operatorname{Ker}\left[G_{1 \ell}\right]$ and $P_{1 \ell}=I-Q_{1 \ell}$.

Theorem 2. Suppose a constant $C_{1}>0$ exists such that the starting values satisfy the relation

$$
\left\|P P_{1 \ell} \mathbf{x}_{\ell}-P P_{1 \ell} \mathbf{x}_{*}\left(t_{\ell}\right)\right\| \leq C_{1} h^{k+1}, \quad \ell<k
$$

and suppose constants $C_{2}$ and $C_{3}$ exits such that

$$
\begin{array}{ll}
\left\|\delta_{\ell}\right\| \leq C_{2} h^{k}, & \ell \geq k, \\
\left\|\hat{\delta}_{\ell}\right\| \leq C_{3} h^{k+1}, & \ell \geq 0 .
\end{array}
$$

Then a constant $C>0$ exists such that

$$
\left\|\mathbf{x}_{*}\left(t_{\ell}\right)-\mathbf{x}_{\ell}\right\| \leq C h^{k}, \quad \ell \geq k .
$$

Outline of the proof (again, for full details see [2]). The structure of the proof of this theorem is similar to the proof of the index- 1 theorem, but more lengthly. With the use of projectors, the DAE is split into an algebraic, an index- 1 and an index- 2 part. We first solve the algebraic equations and use the solution for solving the other parts [2].

Remarks. Circuits which contain L-I cutsets or C-V loops lead to an index2 DAE [12]. For this case, we proved that order reduction for MEBDF occurs (which does not happen for BDF [12]). However the reduction for MEBDF is moderate: the $k$-step MEBDF and the $k$-step BDF, applied to index-2 DAE's, have the same order, namely $k$. However, MEBDF has much better stability properties than BDF [3].

\section{Numerical Tests}

We performed some tests in Matlab with a variable step size fixed order BDF and MEBDF method. We made Work-Precision Diagrams, to compare the work needed for both methods. For more details we refer to [2].

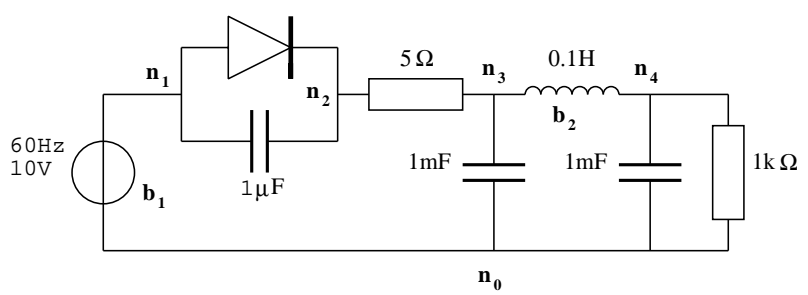

Fig. 1. Rectifier Circuit 


\subsection{Rectifier Circuit}

Fig. 1 shows a rectifier circuit that serves the AC to DC conversion. It is designed in such a way that it damps the incoming sine-wave. We computed a reference solution $\mathbf{x}_{\text {ref }}$ at $t=0.16$ using the BDF method with a very small time step. In Fig. 2(a) we see a Work-Precision Diagram in which we compare the 1-step MEBDF method with the 2-step BDF method. We see that the 1-step MEBDF method even performs slightly better than the 2step BDF. Fig. 2(b) presents a comparison for the 3-step BDF and the 2-step MEBDF, in which the BDF method performs better. Clearly the methods are comparable in these cases, however the MEBDF has the potential of better stability.

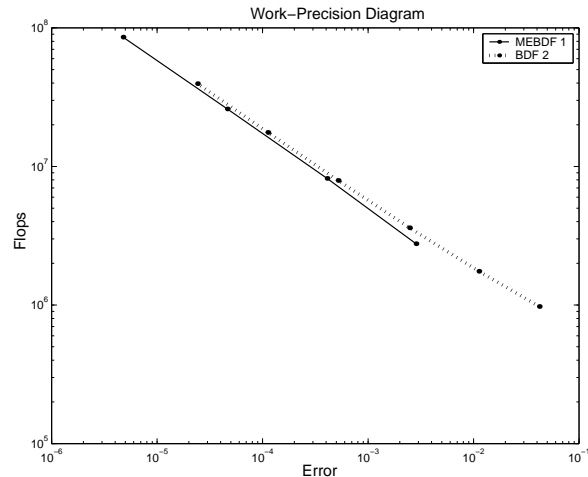

(a)

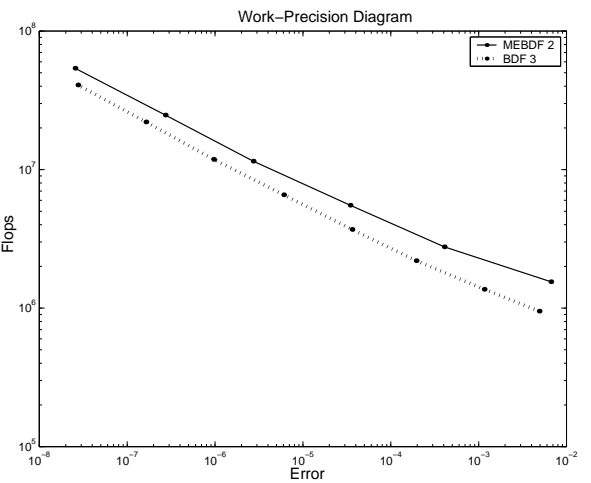

(b)

Fig. 2. Work-Precision Diagram for second order (a) and third order (b) methods

\subsection{Oscillatory Test Problem}

To show how the MEBDF deals with oscillatory problems, we consider an ODE example problem of the form

$$
\begin{aligned}
& x^{\prime}=y+h(r) x, \\
& y^{\prime}=-x+h(r) y,
\end{aligned}
$$

where $r=\sqrt{x^{2}+y^{2}}$. We assume that $0<x_{0}<1,0<y_{0}<1$. The equations can be re-written in the following form:

$$
\begin{aligned}
& r^{\prime}=h(r) r, \\
& \theta^{\prime}=1 .
\end{aligned}
$$


For $h(r)=\frac{1-r}{10}, r=1$ is the only stable limit, $\lim _{t \rightarrow \infty} r(t)=1$. We apply the BDF and MEBDF method to equations (10)-(11), which results in approximate solutions $\left(x_{n}, y_{n}\right)(n=0,1, \ldots)$. We compute $r_{n}=\sqrt{x_{n}^{2}+y_{n}^{2}}$ and plot this as function of $t$. In Fig. 3(a) we did this for the 1-step MEBDF and the 2-step BDF. We see that the MEBDF solution is less damped than the BDF solution of the same order. In Fig.3(b) we see that the 3-step BDF does not perform very well on this problem. This is due to the fact that the 3 -step BDF is not A-stable and for oscillatory problems only A-stable methods perform well. Clearly, the MEBDF method is more suited for oscillatory

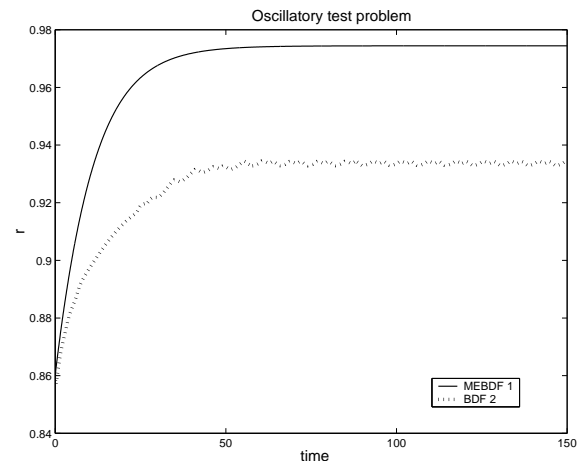

(a)

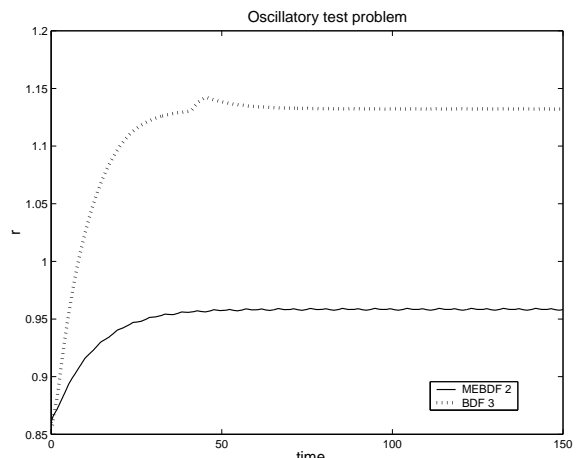

(b)

Fig. 3. Plot of $r_{n}=\sqrt{x_{n}^{2}+y_{n}^{2}}$ by second order (a) and third order (b) methods.

problems than BDF. Here one can also fully exploit the better stability and convergence order properties of MEBDF.

\section{Consistent Solutions}

BDF generates consistent solutions, even if one starts with not consistent initial values $[2,12]$. Because $\mathrm{BDF}$ serves in predicting for the extrapolation point, MEBDF inherits this property from BDF. This is in contrast to for instance solutions obtained by using the Trapezoidal Rule. This property is important when visualizing results for problems with discontinuities. It is known that computing consistent initial values from the DC operating point only requires solving an additional linear system [5]. Another way of getting consistent initial values is integrating forward, till there is no influence of the (inconsistent) initial values and then integrating backward. 


\section{Conclusion}

MEBDF methods offer an alternative to BDF methods in the case of DAE's with index not exceeding 2 . The MEBDF methods combine higher convergence order with better stability properties and better numerical damping: Hence, more rigorous benchmark tests for circuits occurring in practice are worth to be done.

Finally, we gratefully acknowledge discussions, including assistance in providing implementation, with Jeff Cash (Imperial College, London), Jason Frank (CWI, Amsterdam) and Caren Tischendorf (Von Humboldt Universität, Berlin).

\section{References}

1. Brenan, K.E., Campbell, S.L., Petzold, L.R.: Numerical solution of initial-value problems in differential-algebraic equations, SIAM - Society for Industrial and Applied Mathematics, Philadelphia, PA, USA (1996).

2. Bruin, S.M.A.: Modified Extended BDF applied to circuit equations. Philips Research Laboratories, Nat.Lab. Unclassified Report 2001/826, MSc-Thesis Free University Amsterdam (2001). http://www.extra.research.philips.com/publ/rep/nl-ur/ur2001-826.pdf

3. Cash, J.R.: The integration of stiff initial value problems in ODEs using Modified Extended Backward Differentiation Formulae, Comp. \& Maths. with Appls, Vol 9, No 5, (1983) pp. 645-657.

4. Cash, J.R.: Modified extended backward differentiation formulae for the numerical solution of stiff initial value problems in ODEs and DAEs, Journal of Computational and Applied Mathematics, 125, (2000) pp. 117-130.

5. Estévez Schwarz, D., Tischendorf, C.: Structural analysis of electric circuits and consequences for MNA, Int. J. of Circuit Theory and Applications 28, (2000) pp. 131-162.

6. Frank, J.E., van der Houwen, P.J.: Diagonalizable extended backward differential formulas, BIT, Vol 40, No 3, (2000) pp. 497-512.

7. Guenther, M., Rentrop, P., Feldmann, U.: CHORAL - A One Step Method as Numerical Low Pass Filter in Electrical Network Analysis. In: van Rienen, U., Günther M., Hecht D. (Eds.): Scientific Computing in Electrical Engineering, Lecture Notes in Computational Science and Engineering, No 18, (SCEE-2000, Warnemünde), (2001) pp. 199-215.

8. Hairer, E., Nørsett, S.P., Wanner, G.: Solving Ordinary Differential Equations I, Springer Berlin (1987).

9. Hairer, E., Nørsett, S.P., Wanner, G.: Solving Ordinary Differential Equations II, Springer Berlin (1996).

10. Hosea, M.E., Shampine, L.F.: Analysis and implementation of TR-BDF2, Applied Numerical Mathematics 20, (1996) pp. 21-37.

11. de Swart, J.J.B.: Parallel software for implicit differential equations, PhDThesis Univ. of Amsterdam (1997).

12. Tischendorf, C.: Solution of index-2 differential algebraic equations and its application in circuit simulation, Logos Verlag Berlin, PhD-Thesis Humboldt Univ. zu Berlin (1996). 